\title{
Detailed statistical analysis plan for the SafeBoosC III trial: a multinational randomised clinical trial assessing treatment guided by cerebral oxygenation monitoring versus treatment as usual in extremely preterm infants
}

Mathias Lühr Hansen ${ }^{1 *}$ D, Adelina Pellicer ${ }^{2}$, Christian Gluud ${ }^{3}$, Eugene Dempsey ${ }^{4}$, Jonathan Mintzer ${ }^{5}$, Simon Hyttel-Sorensen ${ }^{1}$, Anne Marie Heuchan ${ }^{6}$, Cornelia Hagmann ${ }^{7}$, Gabriel Dimitriou ${ }^{8}$, Gerhard Pichler ${ }^{9}$, Gunnar Naulaers $^{10}$, Guoqiang Cheng ${ }^{11}$, Ana Vilan ${ }^{12}$, Jakub Tkaczyk ${ }^{13}$, Karen B. Kreutzer ${ }^{14}$, Monica Fumagalli ${ }^{15,16}$, Olivier Claris ${ }^{17}$, Siv Fredly ${ }^{18}$, Tomasz Szczapa ${ }^{19}$, Theis Lange ${ }^{20,21}$, Janus Christian Jakobsen ${ }^{3,22,23}$ and Gorm Greisen ${ }^{1}$

\begin{abstract}
Background: Infants born extremely preterm are at high risk of dying or suffering from severe brain injuries. Treatment guided by monitoring of cerebral oxygenation may reduce the risk of death and neurologic complications. The SafeBoosC III trial evaluates the effects of treatment guided by cerebral oxygenation monitoring versus treatment as usual. This article describes the detailed statistical analysis plan for the main publication, with the aim to prevent outcome reporting bias and data-driven analyses.
\end{abstract}

Methods/design: The SafeBoosC III trial is an investigator-initiated, randomised, multinational, pragmatic phase III trial with a parallel group structure, designed to investigate the benefits and harms of treatment based on cerebral near-infrared spectroscopy monitoring compared with treatment as usual. Randomisation will be 1:1 stratified for neonatal intensive care unit and gestational age (lower gestational age $(<26$ weeks) compared to higher gestational age ( $\geq 26$ weeks)). The primary outcome is a composite of death or severe brain injury at 36 weeks postmenstrual age. Primary analysis will be made on the intention-to-treat population for all outcomes, using mixed-model logistic regression adjusting for stratification variables. In the primary analysis, the twin intra-class correlation coefficient will not be considered. However, we will perform sensitivity analyses to address this. Our simulation study suggests that the inclusion of multiple births is unlikely to significantly affect our assessment of intervention effects, and therefore we have chosen the analysis where the twin intra-class correlation coefficient will not be considered as the primary analysis.

Discussion: In line with the Declaration of Helsinki and the International Conference on Harmonization Good Clinical Practice guidelines, we have developed and published this statistical analysis plan for the SafeBoosC III trial, prior to any data analysis.

(Continued on next page)

\footnotetext{
* Correspondence: mathias.safeboosc@gmail.com

1 Department of Neonatology, Rigshospitalet, Copenhagen University

Hospital, Blegdamsvej 9, 2100 Copenhagen, Denmark

Full list of author information is available at the end of the article
}

(c) The Author(s). 2019 Open Access This article is distributed under the terms of the Creative Commons Attribution 4.0 International License (http://creativecommons.org/licenses/by/4.0/), which permits unrestricted use, distribution, and reproduction in any medium, provided you give appropriate credit to the original author(s) and the source, provide a link to the Creative Commons license, and indicate if changes were made. The Creative Commons Public Domain Dedication waiver (http://creativecommons.org/publicdomain/zero/1.0/) applies to the data made available in this article, unless otherwise stated. 
(Continued from previous page)

Trial registration: ClinicalTrials.org, NCT03770741. Registered on 10 December 2018.

Keywords: Randomised clinical trial, Extremely preterm, Near-infrared spectroscopy, Cerebral oximetry, Statistical analysis plan

\section{Background}

Extremely preterm infants carry a high risk of death, with a mortality rate up to $25 \%$ [1, 2]. Furthermore, about $20 \%$ suffer from long-term neurodevelopmental impairment such as cerebral palsy or low intelligence quotient [2, 3]. Psychomotor impairment is a major cause of reduced quality of life and increased costs of medical care, rehabilitation, and special education in this population [4]. Low intelligence quotient affects all aspects of life. With increasing life expectancy, these combined prematurity-related factors pose a significant problem.

Hypoxia has been associated with mortality and brain injury in the preterm population [5]. In the SafeBoosC II trial, cerebral near-infrared spectroscopy (NIRS) monitoring combined with an evidence-based treatment guideline significantly reduced the burden of hypoxia during the first days of life in preterm infants [6]. There were also trends towards reduced occurrence of severe brain injury and mortality [6]. On the other hand, the incidence of bronchopulmonary dysplasia and retinopathy of prematurity was higher among NIRS-monitored neonates [6]. However, SafeBoosC II was not powered to demonstrate effects on these outcomes; thus, high-certainty evidence of clinical benefit and harm in extremely preterm infants is lacking [7]. We therefore plan a larger phase III trial, SafeBoosC III, powered to demonstrate the potential benefits and harms of treatment based on cerebral NIRS monitoring compared with treatment as usual on patient-centred clinical outcomes. As the SafeBoosC III trial will be conducted in compliance with the Declaration of Helsinki in its latest form and the International Conference on Harmonization Good Clinical Practice guidelines [8], we have developed this detailed statistical analysis plan. We believe this will decrease the risk of outcome reporting bias and data-driven analyses.

\section{Methods/design}

\section{Trial overview}

SafeBoosC III is an investigator-initiated, open-label, randomised, multinational, pragmatic phase III clinical trial with a parallel group design. The primary objective is to evaluate the benefits and harms of treatment based on cerebral NIRS monitoring during the first 72 postnatal hours in extremely preterm infants [9], compared with treatment and monitoring as usual, to reduce cerebral hypoxia [10]. The hypothesis is that treatment based on NIRS monitoring for extremely preterm infants during the first $72 \mathrm{~h}$ of life will result in a reduction in death or severe brain injury assessed at 36 weeks postmenstrual age. We plan to test for superiority of the experimental intervention compared with the control group for only the primary outcome, since exploratory outcomes will only be hypothesis generating (see 'Level of significance'). Infants will be randomised with an allocation ratio of 1:1 to either the experimental group or the control group stratified for neonatal intensive care unit (NICU) and gestational age (lower gestational age ( $<26$ weeks) compared to higher gestational age ( $\geq 26$ weeks)). Details of the randomisation method are held securely in the statistics master file. Infants in the experimental group will start cerebral NIRS monitoring as close to birth as possible, but at least within $6 \mathrm{~h}$ of birth, and receive treatment based on NIRS monitoring during the first $72 \mathrm{~h}$ of life (Fig. 1). These treatments will follow an evidence-based treatment guideline [11]. Infants in the control group will not receive cerebral NIRS monitoring and will be monitored and treated according to local guidelines and practices (i.e. treatment as usual). Due to the nature of NIRS, it is difficult to blind the clinical staff or the parents of the trial participants.

Three different consent methods may be used in this trial: prior informed consent (prenatal and postnatal); deferred consent; and prior assent/'opt-out'. The trial will be conducted at more than 50 centres across up to 20 countries (16 European countries, India, China, and the USA), and the protocol will be published in an international peer-reviewed journal [10].

The SafeBoosC III trial is registered at ClinicalTrials.org (NCT03770741) and is compliant with the Declaration of Helsinki in its latest form and with the International Conference on Harmonization Good Clinical Practice. The trial will be approved by relevant authorities, including research ethics boards and data protection agencies, in all participating centres. The progression of the trial can be followed at www.safeboosc.eu. This statistical analysis plan has been written and submitted before randomisation commences and all data analysis for the main publication will be compliant to this plan.

\section{Outcomes}

\section{Primary outcome}

The primary outcome is a composite of either death or severe brain injury. Severe brain injuries will be defined as grade III or IV cerebral haemorrhage (Papile's 


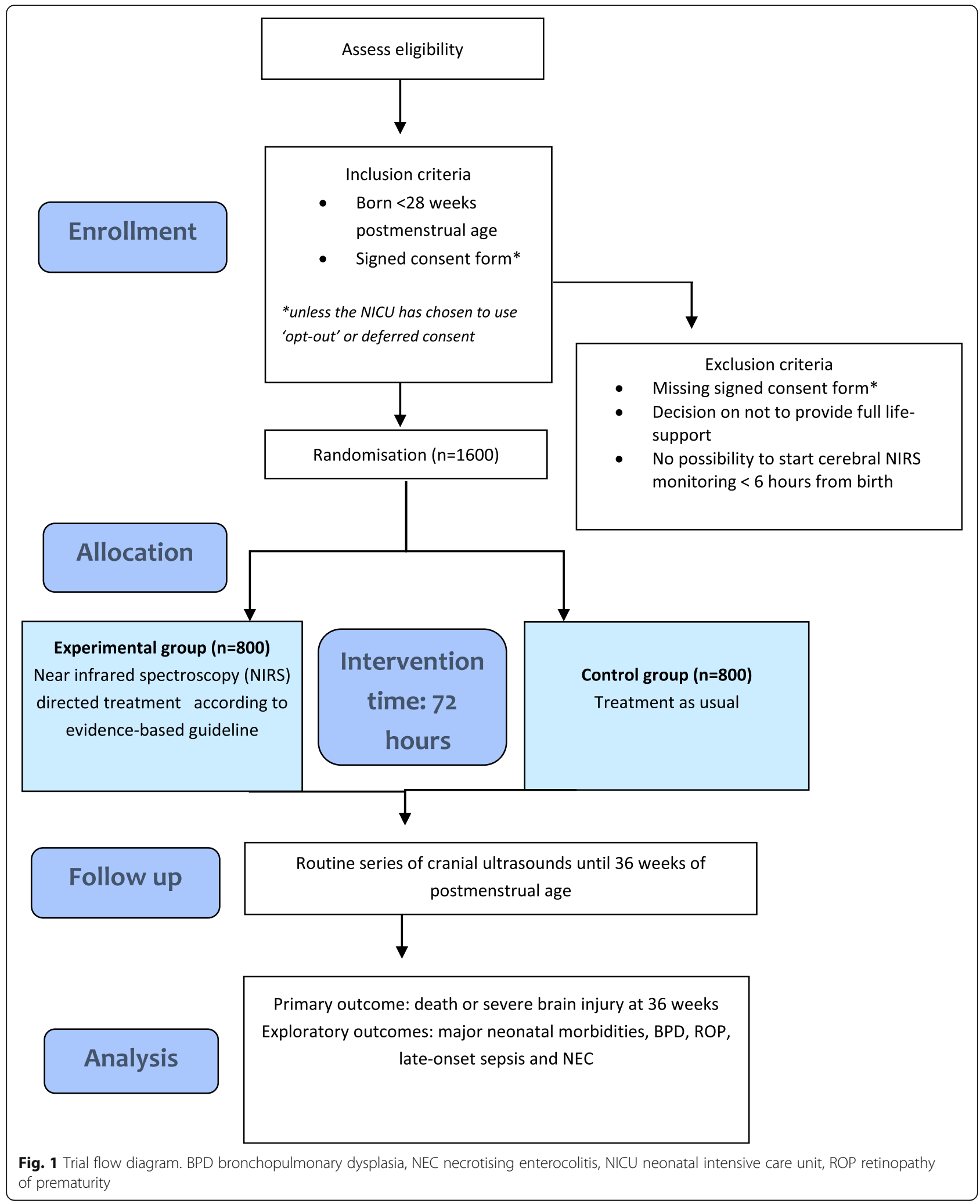

classification) [12], cystic periventricular leukomalacia [2], cerebellar haemorrhage, post-haemorrhagic ventricular dilatation, or cerebral atrophy. These cerebral outcomes will be reported as detected on any one of a series of cranial ultrasound scans that are routinely performed in these infants. 
Outcome assessment of mortality will not be blinded, but diagnosis and classification of brain injury and entry of this information into electronic case report forms will be conducted by a clinician blinded to group allocation.

\section{Exploratory outcomes}

- A count of the presence of the three major neonatal morbidities associated with neurodevelopmental impairment later in life [13]: bronchopulmonary dysplasia (defined below), retinopathy of prematurity (as defined below), and severe brain injury as defined in the primary outcome (i.e. a value of 0,1 , 2, or 3)

- Bronchopulmonary dysplasia defined as oxygen or ventilator/continuous positive airway pressure requirement at 36 weeks' postmenstrual age

- Retinopathy of prematurity stage 3 and above at any time prior to 36 weeks' postmenstrual age

- Late-onset sepsis ( $>72 \mathrm{~h}$ after birth) defined as treatment with antibiotics for at least 5 days

- Necrotising enterocolitis stage 2 or higher using the modified Bell's staging system [14] and/or focal intestinal perforation at any time up until 36 weeks' postmenstrual age

\section{Outcome assessment time point}

All outcomes will be assessed at 36 weeks postmenstrual age.

\section{Sample size}

We have calculated our sample size with an $\alpha$ of $5 \%$, a power of $90 \%$, and a ratio of experimental trial participants to control trial participants of 1:1. The primary outcome is the composite outcome of death or severe brain injury. Sample size calculations were performed for the composite outcome and not for the individual components.

Calculated from the 2009 dataset from the EuroNeoNet project [15] the mortality was $33 \%$ and severe intracranial haemorrhage was observed in $15 \%$. In the SafeBoosC II trial, the proportion of trial participants in the control group with the same composite primary outcome was approximately $34 \%$ and in the experimental group was $26 \%$ [6]. Mortality was $24 \%$ in the control group versus $13 \%$ in the experimental group and the proportion of infants with severe brain injury was $23 \%$ versus $13 \%$ [6].

Based on the aforementioned, a total of 1600 infants - 800 infants randomised to the experimental group and 800 infants to the control group - would be required to demonstrate a reduction of the primary outcome from $34.0 \%$ to $26.5 \%$, with an $\alpha$ of $5 \%$ and a power of $90 \%$. This corresponds to a $22 \%$ relative risk reduction or a $7.5 \%$ absolute risk reduction. We consider this a clinically relevant and important benefit, since mortality is of direct patient relevance and since surviving infants with severe brain injury (about 25\%) are at approximately $40 \%$ risk of moderate-to-severe neurodevelopmental impairment [16]. This absolute risk reduction corresponds to a 'number-needed to treat' of 15 infants and, if our null hypothesis is rejected, is likely to influence clinical practice.

\section{Power calculations for exploratory outcomes}

For the exploratory outcomes, we have performed power calculations as presented in Table 1.

Assuming a mean major neonatal morbidity count (bronchopulmonary dysplasia, retinopathy of prematurity, and severe brain injury) of 0.62 among extremely preterm infants [17], with a standard deviation of 0.80 and a relative risk increase or decrease of $20 \%$ in the experimental group, we will be able to detect this difference between the experimental and control group with $87 \%$ power at a $5 \%$ significance level (Table 1 ).

Assuming a $40 \%$ prevalence of bronchopulmonary dysplasia among extremely preterm infants [18] and a relative risk decrease or increase of $20 \%$ in the experimental group, we will be able to detect this difference between the experimental and control group with $89 \%$ power at a $5 \%$ significance level (Table 1).

Assuming a 13\% prevalence of stage 3 and above retinopathy of prematurity among extremely preterm infants and a relative risk decrease or increase of $30 \%$ in the experimental group [7], we will be able to detect this difference between the experimental and control groups with $68 \%$ power at a $5 \%$ significance level (Table 1 ).

Assuming a $40 \%$ prevalence of late-onset sepsis in the control group [1], defined as treatment with antibiotics for at least 5 days, and a $20 \%$ relative risk decrease or increase in the experimental group, we will be able to detect this difference between the experimental and control groups with $91.2 \%$ power at a $5 \%$ significance level (Table 1).

Assuming an 11\% prevalence of stage 2 and 3 necrotising enterocolitis among extremely preterm infants and a $17 \%$ relative risk decrease or increase in the experimental group, as is the estimate from existing trials [7], we will be able to detect this difference between the experimental and control groups with $23 \%$ power at a $5 \%$ significance level (Table 1).

\section{Assessment of outcomes and additional clinical variables}

There will be three time points for data collection: at randomisation (from 0 to $6 \mathrm{~h}$ after birth); at the end of the intervention period (72 h of life); and at 36 weeks postmenstrual age. Data on feasibility will be assessed at randomisation. At the end of the intervention period, 
Table 1 Overview of power calculations for exploratory outcomes

\begin{tabular}{llll}
\hline Outcome & Assumption on prevalence in background population (\%) & Assumption on risk increase or decrease (\%) & Power (\%) \\
\hline Major neonatal morbidities & $0.62(0.8)^{\text {a }}$ & 20 & 87 \\
Bronchopulmonary dysplasia & 40 & 20 & 89 \\
Retinopathy of prematurity & 13 & 30 & 68 \\
Late-onset sepsis & 40 & 20 & 91.2 \\
Necrotising enterocolitis & 11 & 17 & 23 \\
\hline
\end{tabular}

For definition of outcomes, see 'Outcomes'. All power calculations have been made with a $5 \%$ significance level

${ }^{\text {a }}$ resented as mean count (standard deviation)

data collection will primarily reflect cerebral NIRS monitoring and safety parameters. As mentioned, all outcomes will be assessed at 36 weeks postmenstrual age. Severe brain injury diagnosis and classification data will be collected either by neonatologists assessing all cranial ultrasound scans performed up until 36 weeks postmenstrual age or by reading radiologists' descriptions of these scans. This assessment and data entry will be conducted by a person blinded to group allocation. No long-term follow-up has been formally planned. However, we encourage clinical sites to conduct long-term follow-up, and we have therefore developed an appendix in the protocol (see full protocol at www.safeboosc.eu) describing possible outcomes for later follow-up studies and how these could be conducted. Currently, no protocol for such an ancillary study has been developed.

\section{Explanatory variables}

Additional clinical data on trial participants will be drawn from clinical files, in order to compare characteristics between intervention groups. Data will be drawn from clinical records at $72 \mathrm{~h}$ of age and 36 weeks postmenstrual age. These data consist of a subset of explanatory variables, with the majority usually being reported to the neonatal network databases, such as Vermont Oxford Network [19]. These data will be presented in a table in the main publication (see Table 2). Tests of statistical significance will not be undertaken for explanatory variables. Categorical data will be summarised by numbers and percentages. Continuous data will be summarised by mean and standard deviation if normally distributed or by median and interquartile range if nonnormally distributed.

\section{Safety}

We will report the total number of serious adverse reactions, as defined in the protocol [10] for each group, as well as the total number of participants who experienced one or more serious adverse reactions in each group. We will also report the total number of serious adverse events, as defined in the protocol [10] in each group, as well as the number of participants who experienced one or more serious adverse events in each group.

\section{Level of significance}

The thresholds for significance will be assessed according to a 5-point procedure, suggested by Jakobsen et al. [20]. We will calculate and report confidence intervals and exact $p$-values for the primary and exploratory outcomes. All confidence intervals presented will be $95 \%$ and two-sided. A $p$-value of less than 0.05 will be used as the threshold for statistical significance for our primary outcome, since this value was used as the acceptable risk of type I error in our sample size estimation (see 'Sample size') and since we plan to report on only one primary outcome. However, in our interpretation of the results, we will assess any effect of the experimental intervention according to the point estimate taking into consideration the confidence interval as well as intervention effects on other outcomes [21]. All remaining outcome results will only be considered hypothesisgenerating. Since our primary conclusion will be based on one outcome result at one time point, we will limit problems associated with multiple testing, due to multiple outcome comparisons [22].

Secondly, we will calculate and report the Bayes factor [23] for the primary outcome [24]. The Bayes factor is the ratio between the probability of the results given that the null hypothesis $\left(\mathrm{H}_{0}\right)$ is true divided by the probability of the results given that the alternative hypothesis $\left(\mathrm{H}_{\mathrm{A}}\right)$ is true [23]. In the SafeBoosC III trial, the alternative hypothesis is that the treatment effect is the effect that was used for the sample size calculation: a $22 \%$ relative risk reduction in the experimental group. By calculating the Bayes factor, we will be able to interpret the results of the primary outcome in relation to former trial results [6].

Thirdly, Lan-DeMets monitoring boundaries will be used to adjust the threshold for statistical significance at each interim analysis to judge whether the trial should be terminated early [25]. This is done in order to avoid a false rejection of the null hypothesis based on insufficient sample sizes [26]. The trial will not be stopped prematurely due to futility. The fourth step in the five-step procedure by Jakobsen et al., regarding adjustment of $p$ values based on multiple testing of the primary outcome, is not applicable to our trial, since we have a single primary outcome [20]. 
Table 2 Explanatory variables divided by experimental group and control group participants

\begin{tabular}{|c|c|c|}
\hline Variables & Experimental group $(n)$ & Control group $(n)$ \\
\hline \multicolumn{3}{|l|}{ At randomisation } \\
\hline \multicolumn{3}{|l|}{ Birth weight (g) } \\
\hline \multicolumn{3}{|l|}{ Gestational age (weeks) } \\
\hline \multicolumn{3}{|l|}{ Apgar 1 min $(1-10)$} \\
\hline \multicolumn{3}{|l|}{ Apgar 5 min $(1-10)$} \\
\hline \multicolumn{3}{|l|}{ Gender } \\
\hline \multicolumn{3}{|l|}{ Male (\%) } \\
\hline \multicolumn{3}{|l|}{ Female (\%) } \\
\hline \multicolumn{3}{|l|}{ At $72 \mathrm{~h}$ of age } \\
\hline Age when NIRS monitoring started (h) ${ }^{\mathrm{a}}$ & & N/A \\
\hline Stopping NIRS monitoring before end of monitoring period $(\%)^{\mathrm{a}}$ & & N/A \\
\hline \multicolumn{3}{|l|}{ Parents discontinuing trial participation (\%) } \\
\hline Changes in treatment due to cerebral hypoxia (\%) ${ }^{\mathrm{a}}$ & & N/A \\
\hline Registered cardiovascular support treatment $(\%)^{\mathrm{a}}$ & & N/A \\
\hline $\begin{array}{l}\text { Type of NIRS device used } \\
\text { INVOS }(\%) \\
\text { NIRO (\%) } \\
\text { Fore-Sight (\%) } \\
\text { Sensmart (\%) } \\
\text { O3 (\%) } \\
\text { Egos (\%) } \\
\text { Oxyprem (\%) } \\
\text { Other (\%) }\end{array}$ & & N/A \\
\hline Cerebral NIRS monitoring despite being in control group $(\%)^{b}$ & N/A & \\
\hline \multicolumn{3}{|l|}{ Surfactant therapy (\%) } \\
\hline \multicolumn{3}{|l|}{ Severe adverse reactions (\%) } \\
\hline \multicolumn{3}{|l|}{ At 36 weeks postmenstrual age } \\
\hline \multicolumn{3}{|l|}{ Major congenital anomaly (\%) } \\
\hline \multicolumn{3}{|l|}{ Mechanical ventilation (\%) } \\
\hline \multicolumn{3}{|l|}{ Time with mechanical ventilation (days) } \\
\hline \multicolumn{3}{|l|}{ Patent ductus arteriosus (\%) } \\
\hline \multicolumn{3}{|l|}{ Weight (g) } \\
\hline \multicolumn{3}{|l|}{ Early cranial ultrasound scan (\%) } \\
\hline Late cranial ultrasound scan (\%) & & \\
\hline
\end{tabular}

Data expressed as median (range) for continuous variables, and numbers (percentage) for dichotomous variables

N/A not applicable, NIRS near-infrared spectroscopy

${ }^{2}$ Variables only relevant for experimental group participants

bariables only relevant for control group participants

We will take the upper and lower limits of the confidence intervals into consideration when making study conclusions [21]. Clinical significance will be assessed by calculating the number needed to treat based on the absolute risk reduction data. Based on the results from the phase II trial, we expect an absolute risk reduction of $7.5 \%$, which corresponds to a number needed to treat of 15 (see 'Sample size') [6].

\section{Interim analyses}

One pre-planned interim analysis will be conducted after one-third of trial participants have been randomised. The timing and prevalence of additional interim analyses will be decided solely by the data monitoring and safety committee members. The data monitoring and safety committee will make recommendations to the steering group to continue, change, hold, or terminate the trial. This recommendation will be based primarily on safety considerations and will be guided by statistical monitoring guidelines, defined in the data monitoring and safety committee charter. The data monitoring and safety committee will be provided with the following data from the Coordinating Data Centre: number of participants randomised, number of participants per intervention group $(0,1)$, number of participants stratified per stratification variable per intervention group $(0,1)$, and number of events (primary outcome, SAEs, and SARs) in the two groups. Based on the evaluations of these outcomes, the 
data monitoring and safety committee will decide whether they want further data from the Coordinating Data Centre, and when next to perform analyses of data. Based on the analyses of the safety variables, the data monitoring and safety committee is suggested to use Lan-DeMets sequential monitoring boundaries, based upon a relative risk increase of $100 \%$ of mortality from $25 \%$ to $50 \%$. For any of the other safety outcomes, the statistical limit to guide its recommendations regarding early termination of the trial for harms is recommended also to be conservative.

\section{Handling of missing data}

Missing data will be minimised by performing repeated monitoring of data entry into electronic case report forms. In this way, we will be able to monitor the extent of missing data and intervene if necessary. Hence, we do not anticipate that there will be any significant number of missing values. However, we will consider using multiple imputation and present best-worst and worst-best case scenarios if it is not valid to ignore missing data according to the standards reported by Jakobsen et al. [27]. When using best-worst and worst-best case scenarios, we will assess the potential range of impact of the missing data for the trial results [27]. In the 'best-worst' case scenario, it is assumed that all patients lost to follow-up in the experimental group have had a beneficial outcome, and all those with missing outcomes in the control group have had a harmful outcome [27]. Conversely, in the 'worst-best' case scenario, it is assumed that all patients who were lost to follow-up in the experimental group have had a harmful outcome, and that all those lost to follow-up in the control group have had a beneficial outcome [27].

As recommended, we will describe reasons why outcome data are missing in the main study manuscript [28]. Furthermore, we will compare explanatory variables between all participants randomised to intervention groups (including those with missing outcomes), and also between participants in the intervention groups, where outcomes are reported. This is done to identify imbalances between groups due to missing outcome data [29].

\section{Twins and their intra-cluster correlation}

In extremely preterm populations, $30 \%$ of births may be twins [6], which poses a potential problem for statistical analyses as the outcomes among pairs of twins are potentially correlated [30]. In the SafeBoosC III trial, multiple birth infants will be randomised as a 'pair' or a 'group' (i.e. all siblings will be allocated to the same intervention group). In centres where only one or two cerebral monitoring devices are available, it may not be possible to include all infants from multiple births. Thus, only one of a pair or only one or two infants of triplets may be included. The sibling(s) enrolled in the trial will be the one(s) born last. In the SafeBoosC II trial, the intra-class correlation coefficient (ICC) of the burden of hypoxia within pairs of twins was negligible. The ICC for various binary outcomes has been estimated in a previous study: ICC for death before discharge was estimated as 0.00 (95\% confidence interval (CI) -0.04 to 0.02 ) and for intraventricular haemorrhage grade 3 or 4 as -0.01 (95\% CI -0.05 to 0.01$)$ [31]. These values correlate to a design effect very close to 1 [31]. Therefore, in the primary analysis, we will analyse twin data as independent observations. However, due to the possibility that the correlation between the primary outcome within multiple births will interfere with the estimation of the treatment effect [32], and particularly the assessment of estimation uncertainty, we will perform a sensitivity analysis, taking this effect into consideration. This sensitivity analysis will be performed using the generalised estimating equation (GEE) approach utilising an exchangeable covariance matrix with site (NICU) and stratification variables as fixed effects. The results of both primary outcome analyses will be presented and discrepancies between the two analyses discussed in the final publication. Furthermore, we will calculate, report, and discuss the ICC for the primary outcome.

\section{Stratification}

We will use site (NICU) and gestational age (lower gestational age $(<26$ weeks) compared to higher gestational age ( $\geq 26$ weeks)) as stratification variables in the randomisation. Analyses for all outcomes will be adjusted for these stratification variables [33-35].

\section{Assessment of underlying statistical assumptions}

For all regression analyses, we will test for major interactions between each covariate and the intervention variable. We will, in turn, include each possible first-order interaction between included covariates and the intervention variable. For each combination, we will test whether the interaction term is significant and assess the effect size. We will only consider that there is evidence of an interaction if the interaction is statistically significant after Bonferroni-adjusted thresholds ( 0.05 divided by number of possible interactions) and if the interaction shows a clinically significant effect. If it is concluded that the interaction is significant, we will be presenting an analysis separately for each (e.g. for each site if there is significant interaction between the trial intervention and 'site') and an overall analysis including the interaction term in the model. 


\section{Assessment of underlying statistical assumptions for dichotomous outcomes}

We will assess whether the deviance divided by the degrees of freedom is significantly larger than 1 to assess for relevant overdispersion, and in this case consider using a maximum likelihood estimate of the dispersion parameter. To avoid analytical problems with either zero events or problems such as all participants dying at a given site, we have only included sites planning to randomise a sufficient number of participants. However, we cannot exclude the risk that some sites might have problems with recruitment. We will, by checking whether the number of participants is larger than 10 (rule of thumb) per site, pool the data from small sites if the number of participants is too low.

\section{Statistical analyses}

Analyses will be made on the intention-to-treat population for all outcomes, since this method maintains baseline comparability of the intervention groups [29]. The intention-to-treat population will include all randomised patients, regardless of missing data, lost to follow-up or adherence to the intervention.

In our primary analysis, we will analyse dichotomous outcomes using mixed-effect logistic regression and count data using mixed-effect linear regression with robust standard errors. In all regression models, 'site' will be included as a random effect. The remaining stratification variables (age and intervention groups) will be included as fixed effects. The sensitivity analysis accounting for the possible correlation between twins is described in 'Twins and their intra-cluster correlation'.

As an additional sensitivity analysis, we will perform a per-protocol analysis, only including participants who had no missing data, were not lost to follow-up, and adhered to the intervention. Adherence to the intervention is defined as continuous cerebral oxygenation monitoring during the first $72 \mathrm{~h}$ of life or until death.

We will, in a secondary analysis, analyse the results using random-effects meta-analysis [36].

All outcomes will be analysed collectively since the follow-up time is identical.

\section{Data management}

The data management plan has been described in the protocol paper [10].

\section{CONSORT flow diagram}

The main publication will include a Consolidated Standards of Reporting of Randomised Trials (CONSORT) flow diagram, following the CONSORT 2010 Statement [37]. This will be used to summarise the number of patients who were randomised, allocated to the experimental and control groups, adhered and unadhered to the intervention, lost to follow-up (including parental and physician withdrawal), randomised and included in the primary analysis, and randomised and excluded from the primary analysis.

\section{Withdrawal}

Parents will be able to withdraw consent at any time during the trial. However, data on participants up until the day of withdrawal will be used and participants will be part of the intention-to-treat population and analysis.

\section{Blinding of statisticians}

All data managers, statisticians, and those drawing conclusions will be blinded to treatment allocation. Two blinded statisticians connected to The Copenhagen Trial Unit will independently perform all statistical analyses and the two statistical reports will be published as supplemental material. Discrepancies between the two reports will be discussed by the Steering Committee of the trial. The two intervention groups will be coded ' $A$ ' and ' $\mathrm{B}$ '. When comparability between the two independent analyses have been obtained, two abstracts will be written: one assuming ' $\mathrm{A}$ ' is the experimental group and ' $\mathrm{B}$ ' is the control group - and one assuming the opposite. After the conclusions have been drawn, blinding will be broken, and the final manuscript will be based on the correct pre-written abstract.

\section{Simulation of twin scenarios}

To explore the potential impact of twin correlation, we conducted a simulation study to assess potential impact on power and coverage probabilities of confidence intervals (i.e. does the computed 95\% CI contain the true parameter values with $95 \%$ probability). We compared the naive analysis (primary analysis of the primary outcome), which ignores twin pairs, to a GEE-based approach which does account for twin correlation. We did this by simulating 10,000 trials with sample size and true parameter values as in the sample size estimation and varied twin probability and ICC. These results are presented in Table 3. This simulation study shows that for a low ICC value or low twin proportion, we can expect both the naive and GEE analyses to have correct coverage and equal power. For a high ICC and a high twin proportion, we can expect the GEE analysis to retain correct coverage, while the naive analysis will have decreased coverage; these differences, however, would be minimal. For high twin proportion and high ICC values, the effective sample size was reduced, which as expected implied that the correct analysis (the GEE) yields a lower power than the intended $90 \%$, albeit only marginally so, and that the coverage for the naïve analysis was a bit too low. 


\section{Discussion}

This article presents the detailed statistical analysis plan for the SafeBoosC phase III trial. It has been developed and submitted prior to any randomisation or data collection in order to avoid data-driven analyses and outcome reporting bias. Data will be analysed on the intention-totreat population, and multiple imputations will be used if the proportion of missing data cannot be ignored (see 'Handling of missing data'). An anonymised dataset regarding all outcomes will be uploaded to a public database to be available for other researchers and peers 6 months after acceptance of the study manuscript.

We plan to report on both primary and exploratory outcomes in the main publication, but the conclusion will solely be based on the results of the primary outcome. If the result is statistically insignificant, based on the 5-point procedure by Jakobsen et al. [20], we will conclude that there is no significant difference between the intervention and treatment as usual (see 'Level of significance').

\section{Dealing with multiple analyses}

Planning multiple analyses on a primary outcome has the potential to increase the risk of type I errors, due to multiple testing [38]. If it is predefined that a significant difference between the experimental and control groups on any one of the primary outcome analyses is sufficient to declare superiority of a given intervention, one would have to correct for multiple testing by decreasing the $\alpha$ value $[22,39]$. On the other hand, planning that all primary outcome analyses must show significant benefit of the intervention to declare superiority has the potential to increase the risk of type II errors, due to insufficiently powered analyses [40]. Hence, by only planning one analysis for the primary outcome and by defining additional analyses as sensitivity analyses (see 'Twins and their intra-cluster correlation'), we have eliminated the type I and type II error-related issues described above. The sensitivity analyses on the primary outcome will only be used to discuss and illustrate the results of the primary analysis.

\section{Strengths}

According to our knowledge, SafeBoosC III will provide the largest trial, thus far, evaluating the benefits and harms of treatment guided by cerebral NIRS monitoring - not only in extremely preterm infants [41] but across all patient populations [42].

It is an important strength that both the protocol and statistical analysis plan have been developed and submitted prior to any randomisation or data collection $[8,10]$. Furthermore, we have also taken the issue of twins and their intra-cluster correlation into consideration, by

Table 3 Simulation study to assess power and coverage probabilities of confidence intervals of primary outcome

\begin{tabular}{|c|c|c|c|c|c|}
\hline ICC & $\begin{array}{l}\text { Proportion of } \\
\text { twins }\end{array}$ & $\begin{array}{l}\text { Power of naive } \\
\text { analysis }\end{array}$ & $\begin{array}{l}\text { Power of GEE } \\
\text { analysis }\end{array}$ & $\begin{array}{l}\text { Coverage probability of naive } \\
\text { analysis }\end{array}$ & $\begin{array}{l}\text { Coverage probability of GEE } \\
\text { analysis }\end{array}$ \\
\hline 0 & 0.1 & 0.91 & 0.91 & 0.95 & 0.95 \\
\hline 0 & 0.2 & 0.91 & 0.91 & 0.95 & 0.95 \\
\hline 0 & 0.3 & 0.90 & 0.90 & 0.95 & 0.95 \\
\hline 0 & 0.4 & 0.90 & 0.90 & 0.95 & 0.95 \\
\hline 0.01 & 0.1 & 0.91 & 0.91 & 0.95 & 0.95 \\
\hline 0.01 & 0.2 & 0.90 & 0.90 & 0.95 & 0.95 \\
\hline 0.01 & 0.3 & 0.90 & 0.90 & 0.95 & 0.95 \\
\hline 0.01 & 0.4 & 0.91 & 0.90 & 0.95 & 0.95 \\
\hline 0.03 & 0.1 & 0.90 & 0.90 & 0.95 & 0.95 \\
\hline 0.03 & 0.2 & 0.91 & 0.91 & 0.95 & 0.95 \\
\hline 0.03 & 0.3 & 0.90 & 0.90 & 0.95 & 0.95 \\
\hline 0.03 & 0.4 & 0.90 & 0.90 & 0.95 & 0.95 \\
\hline 0.13 & 0.1 & 0.90 & 0.90 & 0.95 & 0.95 \\
\hline 0.13 & 0.2 & 0.90 & 0.89 & 0.94 & 0.95 \\
\hline 0.13 & 0.3 & 0.90 & 0.89 & 0.94 & 0.95 \\
\hline 0.13 & 0.4 & 0.90 & 0.88 & 0.94 & 0.95 \\
\hline 0.2 & 0.1 & 0.90 & 0.90 & 0.95 & 0.95 \\
\hline 0.2 & 0.2 & 0.90 & 0.89 & 0.94 & 0.95 \\
\hline 0.2 & 0.3 & 0.90 & 0.88 & 0.94 & 0.95 \\
\hline 0.2 & 0.4 & 0.89 & 0.87 & 0.94 & 0.95 \\
\hline
\end{tabular}

GEE generalised estimating equation, ICC intra-class coefficient 
performing an additional sensitivity analysis to address its potential effect on results (see section on 'Twins and their intra-cluster correlation'). To address the potential impact of twin correlation on our results, we also performed a simulation study, showing that we can expect the potential impact of twin correlation to be minor.

There is genuine evidence that most randomised clinical trials lack external validity, which is an important explanation for why multiple interventions proven beneficial in randomised clinical trials are underused in routine clinical practice [43]. Since SafeBoosC III is an international trial including multiple sites across different countries, limitations to external validity such as different practices between countries and health-care systems seems less of an issue for external validity. Furthermore, the external validity of our results will also be described in the main publication, as recommended in the Consolidated Standards of Reporting of Randomised Trials guidelines [37].

\section{Limitations}

Our methodology also has limitations. Only three of the five exploratory outcomes are sufficiently powered $(80 \%$ power) to show a significant difference between the experimental and control groups, at a 5\% significance level. If these were categorised as secondary or additional primary outcomes, we would need to correct for multiple testing by decreasing the $\alpha$ value using Bonferroni adjustments [39]. Therefore, we will not make any clinical conclusions based on these results. However, we believe they are important to report and assess since they represent major neonatal morbidities in our study population $[1,13,44]$.

As thoroughly reported in the SafeBoosC III design paper [10], it is difficult to blind clinical staff, the infant, and the parents of the trial participants, which introduces risks of bias [45-48]. This important concern is discussed in detail in our design paper [10].

As recommended in the European Medicines Agency Guidelines on Multiplicity Issues in Clinical Trials, the components of the primary composite outcome (i.e. death and severe brain injury) will be analysed separately [49]. However, interpretation of these sub-analyses will be difficult, since death and severe brain injury as individual outcomes are insufficiently powered to show a real benefit of the intervention.

\section{Trial status}

At present, the study protocol has been registered at www.clinicaltrials.gov (NCT 03770741, registered on 10 December 2018) and has been accepted for publication [10]. The first participant was randomised on 27 June 2019. Status on recruitment can be accessed at www. safeboosc.eu.

\section{Statistical analysis plan status}

Version 1.0 (8 August 2019). This document has been written based on information available in the protocol paper [10].

\section{Abbreviations}

Cl: Confidence interval; GEE: Generalised estimating equation; ICC: Intra-class correlation coefficient; NICU: Neonatal intensive care unit; NIRS: Near-infrared spectroscopy

\section{Acknowledgements}

The authors thank the trial participants in the SafeBoosC II trial for providing information for the design of the present SafeBoosC III trial. They thank the Elsass Foundation for monetary support.

\section{Roles and responsibilities}

MLH has taken the lead in writing the statistical analysis plan. JCJ is the senior statistician. GG is the coordinating investigator.

\section{Authors' contributions}

MLH, GG, JCJ, CG, and TL contributed to the conception and design of the statistical analysis plan and drafted the main manuscript. $\mathrm{AMH}, \mathrm{AP}, \mathrm{AV}, \mathrm{CH}$, ED, GD, GP, GN, GC, JM, JT, KBK, MF, OC, SH-S, SF, and TS all made substantial contributions to the process of developing the manuscript and revised the manuscript critically for important intellectual content. All authors will give final approval of the version to be published.

\section{Funding}

The sponsor/coordinating investigator, Professor of Neonatology Gorm Greisen, is the initiator of the SafeBoosC III project. He has no financial interest in the results of the trial nor in the NIRS devices. The Elsass Foundation supported this work through an unconditional and unrestricted grant of DKK 2,700,000, Svend Andersen foundation with an unconditional and unrestricted grant of DKK 1,000,000 and Aage og Louis-Hansens foundation with an unconditional and unrestricted grant of DKK 1,000,000. These funding sources had no role in the design of this study and will not have any role during its execution, analyses, interpretation of the data, or decision to submit results. As we seek additional local and central funding, including funding from industry sponsors, such sources will not influence the methodology, data, analysis, reporting, or conclusions of the trial. Furthermore, any participating department can seek local/national support from all sources, including dealers of devices, as long as such sources will not influence the methodology, data, analysis, reporting, or conclusions of the trial.

\section{Availability of data and materials}

Not applicable.

\section{Ethics approval and consent to participate}

By 15 March 2019, the trial had been approved in 55 neonatal intensive care units in Austria, China, Czech Republic, Denmark, Greece, Ireland, Italy, Norway, Poland, Portugal, Spain, Switzerland and Turkey. No sites will start randomising participants before ethics approval has been granted. Status on ethical approval for all participating sites can be found at www.safeboosc.eu. Written informed consent will be obtained by a qualified physician or nurse connected to the trial prior to randomisation of any participant, unless the neonatal intensive care unit (NICU) uses deferred informed consent or prior assent as consent methods. These consent procedures will be approved by local ethics committees or institutional review boards before use.

Consent for publication

Not applicable.

\section{Competing interests}

The authors declare that they have no competing interests.

\section{Author details}

'Department of Neonatology, Rigshospitalet, Copenhagen University Hospital, Blegdamsvej 9, 2100 Copenhagen, Denmark. ²Department of Neonatology, La Paz University Hospital, Paseo De La Castellana 261, 28046 Madrid, Spain. ${ }^{3}$ Copenhagen Trial Unit, Centre for Clinical Intervention 
Research, Rigshospitalet, Copenhagen University Hospital, Blegdamsvej 9, 2100 Copenhagen, Denmark. ${ }^{4}$ Infant Centre and Department of Paediatrics and Child Health, University College Cork, College Road, Cork, Ireland. ${ }^{5}$ Division of Neonatal-Perinatal Medicine, Mountainside Medical Center, Montclair, NJ, USA. 'Department of Neonatology, Royal Hospital for Children, 1345 Govan Rd, Glasgow G51 4TF, UK. ${ }^{7}$ Department of Neonatology, Children's University Hospital of Zürich, Steinweisstrasse 75, 8037 Zurich, Switzerland. ${ }^{8} \mathrm{NICU}$, Department of Pediatrics, University General Hospital of Patras, 26504 Patras, Greece. ${ }^{9}$ Department of Pediatrics, Medical University of Graz, Auenbruggerplatz 30, Graz, Austria. ${ }^{10}$ Department of Neonatology, University Hospital Leuven, Herestraat 49, Leuven, Belgium. ${ }^{11}$ Department of Neonatology, Children's Hospital of Fudan University, 399 Wanyuan Rd, Minhang Qu, Shanghai Shi, China. ${ }^{12}$ Department of Neonatology, Centrohospitalar Universitário de São João, Alameda Prof. Hernâni Monteiro, 4200-319 Porto, Portugal. ${ }^{13}$ Department of Neonatology, University Hospital Motol, V Uvalu 84, 15006 Prague 5, Czech Republic. ${ }^{14}$ Department of Neonatology, University Children's Hospital Tuebingen, Hoppe-Seyler-Straße 1, 72076 Tuebingen, Germany. ${ }^{15}$ Fondazione IRCCS Cà Granda Ospedale Maggiore Policlinico Milan, Via della Commenda 12, 20122 Milan, Italy.

${ }^{16}$ Department of Clinical Sciences and Community Health, University of Milan, Milan, Italy. ${ }^{17}$ Department of Neonatology, Hospices Civil De Lyon, 3 Quai des Célestins, 69002 Lyon, France. ${ }^{18}$ Department of Neonatology, Oslo University Hospital, Kirkeveien, 1660450 Oslo, Norway. ${ }^{19}$ Department of Neonatology, Poznan University of Medical Sciences, Polna 33, 60-535 Poznań, Poland. ${ }^{20}$ Section of Biostatistics, Department of Publich Health, Copenhagen University, Øster Farimagsgade 5, Copenhagen K, Denmark. ${ }^{21}$ Center for Statistical Science, Peking University, Beijing, China.

${ }^{22}$ Department of Cardiology, Holbæk Hospital, Smedelundsgade 60, 4300 Holbæk, Denmark. ${ }^{23}$ Department of Regional Health Research, The Faculty of Health Sciences, University of Southern Denmark, Odense, Denmark.

\section{Received: 2 May 2019 Accepted: 25 September 2019}

\section{Published online: 19 December 2019}

\section{References}

1. Stoll BJ, Hansen NI, Bell EF, Shankaran S, Laptook AR, Walsh MC, et al. Neonatal outcomes of extremely preterm infants from the NICHD neonatal research network. Pediatrics. 2010;126:443-56.

2. Volpe JJ. Brain injury in the premature infant: overview of clinical aspects, neuropathology, and pathogenesis. Semin Pediatr Neurol. 1998;5:135-51.

3. Adams-Chapman I, Heyne RJ, DeMauro SB, Duncan AF, Hintz SR, Pappas A, et al. Neurodevelopmental impairment among extremely preterm infants in the neonatal research network. Pediatrics. 2018;141:e20173091.

4. Behrman R, Butler AS. Preterm birth: causes, consequences and prevention. Institute of Medicine. Washington, DC: National Academies Press; 2007.

5. Volpe JJ. Brain injury in premature infants: a complex amalgam of destructive and developmental disturbances. Lancet Neurol. 2009;8:110-24.

6. Hyttel-Sørensen S, Pellicer A, Alderliesten T, Austin T, Van Bel F, Benders M, et al. Cerebral near infrared spectroscopy oximetry in extremely preterm infants: phase II randomised clinical trial. BMJ. 2015;350:1-11.

7. Hyttel-Sørensen S, Greisen G, Als-Nielsen B, Gluud C. Cerebral near-infrared spectroscopy monitoring for prevention of brain injury in very preterm infants. Cochrane Database Syst Rev. 2017;9:CD011506.

8. Guideline on Good Clinical Practice E6(R2). London: European Medicines Agency, Committee for Human Medicinal Products. 2017.

9. World Health Organization. Preterm birth. https:/www.who.int/news-room/ fact-sheets/detail/preterm-birth. 2018. Accessed 24 Nov 2019.

10. Hansen ML, Pellicer A, Gluud C, Dempsey E, Mintzer J, Hyttel-Sørensen S, et al. Cerebral near-infrared spectroscopy monitoring versus treatment as usual for extremely preterm infants. A protocol for the SafeBoosC phase III randomized clinical trial. Manuscript accepted for publication in Trials. 2019

11. Pellicer A, Greisen G, Benders M, Claris O, Dempsey E, Fumagally M, et al. The SafeBoosC phase II randomised clinical trial: a treatment guideline for targeted near-infrared-derived cerebral tissue oxygenation versus standard treatment in extremely preterm infants. Neonatology. 2013;104:171-8.

12. Papile LA, Burstein J, Burstein $\mathrm{R}$, Koffler $\mathrm{H}$. Incidence and evolution of subependymal and intraventricular hemorrhage: a study of infants with birth weights less than 1,500 gm. J Pediatr. 1978;92:529-34.

13. Holsti A, Serenius F, Farooqi A. Impact of major neonatal morbidities on adolescents born at 23-25 weeks of gestation. Acta Paediatr. 2018;107: 1893-901.
14. Bell MJ, Ternberg JL, Feigin RD, Keating JP, Marshall R, Barton L, et al. Neonatal necrotizing enterocolitis. Therapeutic decisions based upon clinical staging. Ann Surg. 1978;187:1-7.

15. Valls-I-Soler A, Carnielli V, Claris O, de la Cruz Bértolo J, Halliday HL, Hallman M, Hummler H, Weindling M; Scientific Steering Committee von EuroNeoStat (siehe Anhang). [EuroNeoStat: a European information system on the outcomes of care for very-low-birth-weight infants (< $1500 \mathrm{~g})]$. Z Geburtshilfe Neonatol. 2008;212(3):116-8. https://doi.org/10.1055/s-20081076802. German. PMID: 18709632.

16. Hintz SR, Barnes PD, Bulas D, Slovis TL, Finer NN, Wrage LA, et al. Neuroimaging and neurodevelopmental outcome in extremely preterm infants. Pediatrics. 2015;135:e32-42.

17. Schmidt B, Roberts RS, Davis PG, Doyle LW, Asztalos EV, Opie G, et al. Prediction of late death or disability at age 5 years using a count of 3 neonatal morbidities in very low birth weight infants. J Pediatr. 2015;167: 982-986.e2

18. Davidson L, Berkelhamer S. Bronchopulmonary dysplasia: chronic lung disease of infancy and long-term pulmonary outcomes. J Clin Med. 2017;6:4.

19. Vermont Oxford Network. Vermont Oxford Network. 2019. https://public. vtoxford.org/. Accessed 24 Nov 2019.

20. Jakobsen JC, Gluud C, Winkel P, Lange T, Wetterslev J. The thresholds for statistical and clinical significance - a five-step procedure for evaluation of intervention effects in randomised clinical trials. BMC Med Res Methodol. 2014;14:34.

21. Amrhein V, Greenland S, McShane B. Scientists rise up against statistical significance. Nature. 2019;567:305-7.

22. Zhang J, Quan H, Ng J, Stepanavage ME. Some statistical methods for multiple endpoints in clinical trials. Control Clin Trials. 1997;18:204-21.

23. Goodman SN. Introduction to Bayesian methods I: measuring the strength of evidence. Clin Trials J Soc Clin Trials. 2005;2:282-90.

24. Wasserstein RL, Schirm AL, Lazar NA. Moving to a world beyond " $p<0.05$ ". Am Stat. 2019;73:1-19.

25. DeMets DL, Lan KKG. Interim analysis: the alpha spending function approach. Stat Med. 1994;13:1341-52.

26. Guyatt GH, Briel M, Glasziou P, Bassler D, Montori VM. Problems of stopping trials early. BMJ. 2012;344:e3863.

27. Jakobsen JC, Gluud C, Wetterslev J, Winkel P. When and how should multiple imputation be used for handling missing data in randomised clinical trials - a practical guide with flowcharts. BMC Med Res Methodol. 2017:17:162

28. Little RJ, D'Agostino R, Cohen ML, Dickersin K, Emerson SS, Farrar JT, et al. The prevention and treatment of missing data in clinical trials. $\mathrm{N} \mathrm{Engl} \mathrm{J}$ Med. 2012;367:1355-60

29. Groenwold RHH, Moons KGM, Vandenbroucke JP. Randomized trials with missing outcome data: how to analyze and what to report. Can Med Assoc J. 2014;186:1153-7.

30. Gates S, Brocklehurst P. How should randomised trials including multiple pregnancies be analysed? BJOG An Int J Obstet Gynaecol. 2004;111:213-9.

31. Yelland LN, Sullivan TR, Collins CT, Price DJ, McPhee AJ, Lee KJ. Accounting for twin births in sample size calculations for randomised trials. Paediatr Perinat Epidemiol. 2018;32:380-7.

32. Shaffer ML, Hiriote $\mathrm{S}$. Analysis of time-to-event and duration outcomes in neonatal clinical trials with twin births. Contemp Clin Trials. 2009;30:150-4.

33. Kahan BC, Morris TP. Reporting and analysis of trials using stratified randomisation in leading medical journals: review and reanalysis. BMJ. 2012; 345:e5840.

34. Kahan BC, Morris TP. Improper analysis of trials randomised using stratified blocks or minimisation. Stat Med. 2012;31:328-40.

35. Brennan KC. Accounting for centre-effects in multicentre trials with a binary outcome - when, why, and how? BMC Med Res Methodol. 2014;14:1-11.

36. Deeks JJ, Higgins JPT, Altman DG. Chapter 10: analysing data and undertaking meta-analyses. Draft version (29 January 2019) for inclusion. In: Higgins JPT, Thomas J, Chandler J, Cumpston M, Li T, Page MJ, Welch VA, editors. Cochrane handbook for systematic reviews of interventions. London: Cochrane. In Press.

37. Moher D, Hopewell S, Schulz KF, Montori V, Gotzsche PC, Devereaux PJ, et al. CONSORT 2010 explanation and elaboration: updated guidelines for reporting parallel group randomised trials. BMJ. 2010;340:C869.

38. Li G, Taljaard M, Van den Heuvel ER, Levine MA, Cook DJ, Wells GA, et al. An introduction to multiplicity issues in clinical trials: the what, why, when and how. Int J Epidemiol. 2017;46:746-55. 
39. Abdi H. The Bonferonni and Šidák corrections for multiple comparisons. In Salkind N, editor. Encyclopedia of measurement and statistics. Thousand Oaks: Sage; 2007. p. 1-9.

40. Akobeng AK. Understanding type I and type || errors, statistical power and sample size. Acta Paediatr. 2016;105:605-60.

41. Yu Y, Zhang K, Zhang L, Zong H, Meng L, Han R. Cerebral near-infrared spectroscopy (NIRS) for perioperative monitoring of brain oxygenation in children and adults. Cochrane Database Syst Rev. 2018;1:CD010947.

42. Taillefer MC, Denault AY. Cerebral near-infrared spectroscopy in adult heart surgery: systematic review of its clinical efficacy. Can J Anaesth. 2005;52:79-87.

43. Rothwell PM. External validity of randomised controlled trials: "To whom do the results of this trial apply?". Lancet. 2005;365:82-93.

44. Ward RM, Beachy JC. Neonatal complications following preterm birth. BJOG An Int J Obstet Gynaecol. 2003:110:8-16.

45. Savović J, Turner RM, Mawdsley D, Jones HE, Beynon R, Higgins JPT, et al. Association between risk-of-bias assessments and results of randomized trials in Cochrane Reviews: the ROBES Meta-Epidemiologic Study. Am J Epidemiol. 2018;187:1113-22.

46. Hrobjartsson A, Thomsen ASS, Emanuelsson F, Tendal B, Hilden J, Boutron I, et al. Observer bias in randomized clinical trials with measurement scale outcomes: a systematic review of trials with both blinded and nonblinded assessors. Can Med Assoc J. 2013;185:E201-11.

47. Hróbjartsson A, Thomsen ASS, Emanuelsson F, Tendal B, Rasmussen JV, Hilden J, et al. Observer bias in randomized clinical trials with time-to-event outcomes: systematic review of trials with both blinded and non-blinded outcome assessors. Int J Epidemiol. 2014;43:937-48.

48. Hróbjartsson A, Emanuelsson F, Skou Thomsen AS, Hilden J, Brorson S. Bias due to lack of patient blinding in clinical trials. A systematic review of trials randomizing patients to blind and nonblind sub-studies. Int J Epidemiol. 2014:43:1272-83

49. EMA (European Medicines Agency). Guideline on multiplicity issues in clinical trials. 2017.

\section{Publisher's Note}

Springer Nature remains neutral with regard to jurisdictional claims in published maps and institutional affiliations.

Ready to submit your research? Choose BMC and benefit from:

- fast, convenient online submission

- thorough peer review by experienced researchers in your field

- rapid publication on acceptance

- support for research data, including large and complex data types

- gold Open Access which fosters wider collaboration and increased citations

- maximum visibility for your research: over $100 \mathrm{M}$ website views per year

At $\mathrm{BMC}$, research is always in progress.

Learn more biomedcentral.com/submissions 